\title{
Editorial
}

\section{Recent Advances in Cellulose-Based Materials: Synthesis, Characterization, and Their Applications}

\author{
Xue-Ming Zhang, ${ }^{1}$ Ming-Guo Ma, ${ }^{1}$ Jun Yang, ${ }^{2}$ Zhouyang Xiang, \\ Jie-Fang $\mathrm{Zhu}^{4}{ }^{4}$ and Yatimah Alias ${ }^{5}$ \\ ${ }^{1}$ Beijing Key Laboratory of Lignocellulosic Chemistry, College of Materials Science and Technology, \\ Beijing Forestry University, Beijing 100083, China \\ ${ }^{2}$ University of British Columbia, Vancouver, BC, Canada \\ ${ }^{3}$ University of Wisconsin-Madison, Madison, WI, USA \\ ${ }^{4}$ Department of Materials Chemistry, Ångström Laboratory, Uppsala University, 75121 Uppsala, Sweden \\ ${ }^{5}$ University of Malaya, Kuala Lumpur, Malaysia
}

Correspondence should be addressed to Xue-Ming Zhang; xm_zhang@bjfu.edu.cn

Received 5 October 2016; Accepted 9 October 2016

Copyright (C) 2016 Xue-Ming Zhang et al. This is an open access article distributed under the Creative Commons Attribution License, which permits unrestricted use, distribution, and reproduction in any medium, provided the original work is properly cited.

Recently, sustainable energy sources and environmental problems have received more attention. As one of the most abundant polysaccharides and renewable resources on the earth, cellulose is widely used in environmental protection, water treatment, biomedical, and other fields owing to its particular characteristics such as mechanical properties, biodegradation, biocompatibility, and low toxicity. More importantly, cellulose could be used as an important resource for the transform to high value-added bioethanol, biomaterials, and biochemicals. Based on its wide applications and excellent properties, this special issue focused on the synthesis and applications of cellulose-based materials and explores the new synthetic methods, characterization analysis techniques, and high value-added applications fields of cellulose materials.

In this special issue, we received 18 papers for submission. Finally, 10 papers were selected for publication. Firstly, we thank the great contributions and enthusiastic support from the authors to the special issue.

Developments of novel cellulose-based materials open up new prospects for cellulose in application of reinforcement, food packaging, and photocatalytic activity. The paper titled "Self-Shrinkage Behaviors of Waste Paper Fiber Reinforced Cement Paste considering Its Self-Curing Effect at EarlyAges" reports self-shrinkage behaviors of waste paper fiber reinforced cement paste and explored its self-curing effect at early ages. The authors discovered that the waste paper fibers could mitigate the self-shrinkage of cement paste under the wet mixing condition, which displayed significant selfshrinkage cracking control while providing some internal curing. In addition, the paper titled "Extraction and Hydrophobic Modification of Cotton Stalk Bark Fiber" reports the extraction and hydrophobic modification cotton stalk bark fiber. These as-modified materials are expected to be a filler with strengthening effect in wood plastic composites fields. Moreover, the synthesis of uniform spherical nanoporous $\mathrm{TiO}_{2}$ aerogel templated by cellulose aerogel has been reported in the paper titled "Synthesis and Characterization of Uniform Spherical Nanoporous $\mathrm{TiO}_{2}$ Aerogel Templated by Cellulose Alcohol-Gel with Enhanced Photocatalytic Activity." It was found that $\mathrm{TiO}_{2}$ aerogels displayed uniform sphere shape, good internal morphology, and high specific surface area of $149.95 \mathrm{~m}^{2} \mathrm{~g}^{-1}$. The authors found that these cellulose aerogels displayed the enhanced photocatalytic activity, which had a high degradation ratio of $92.9 \%$ on methyl orange dye.

The paper titled "Waste Composite Sensor Designed by Cellulose and Activated Carbon as Ethylene Absorber" reports the design of waste composite sensor by cellulose and activated carbon as ethylene absorber. It possessed good performance in specific surface area and porosity after chemical 
modification. More importantly, these materials displayed the increase ethylene gas adsorption for food packaging.

After that, they applied eggshell and bacterial cellulose composite membrane as absorbent material in active packaging, in which the flexibility and thermal stability of composite were enhanced. These composites also displayed good adsorption experiment on water and vegetable oil capacity due to the existence of eggshell in bacterial cellulose composite. Authors indicated that these cellulose-based composites exhibited the applications potential for absorbent material in active packaging.

Ionic liquid is one of excellent solvents for dissolving of cellulose. The paper titled "Homogeneous Modification of Sugarcane Bagasse by Graft Copolymerization in Ionic Liquid for Oil Absorption Application" reports the homogeneous modification of sugarcane bagasse by graft copolymerization in ionic liquid for oil adsorption application. Experiment results indicated that the thermal stability of sugarcane bagasse decreased after the homogeneous modification by the graft copolymerization. The study provides an alternative strategy to convert sugarcane bagasse into a value-added material. H. H. Wang et al. researched the reaction behavior of cellulose in the homogeneous esterification of bagasse modified with phthalic anhydride in ionic liquid 1-allyl-3methylimidazolium chloride. These results provide detailed understanding of the homogenous modification mechanism of lignocellulose.

Moreover, A. B. Morales-Cepeda et al. reported the deposition of ibuprofen crystals on hydroxypropyl cellulose/polyacrylamide gel. K.-H. Tseng et al. combined conductivity method and electric spark discharge method to fabricated bacteriostatic substrate with electrospinning for silver dressing. C. Arancibia et al. investigated the application of $\mathrm{CMC}$ as thickener on nanoemulsions based on olive oil.

As mentioned above in the as-reported papers, this special issue provides the recent development of cellulosebased materials. We expect that the rapid progress and further development of these advanced cellulose-based materials will be achieved in the near future.

\section{Acknowledgments}

We appreciate the great efforts and contributions from the authors and reviewers to the special issue.

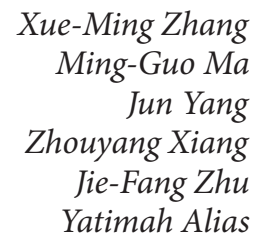



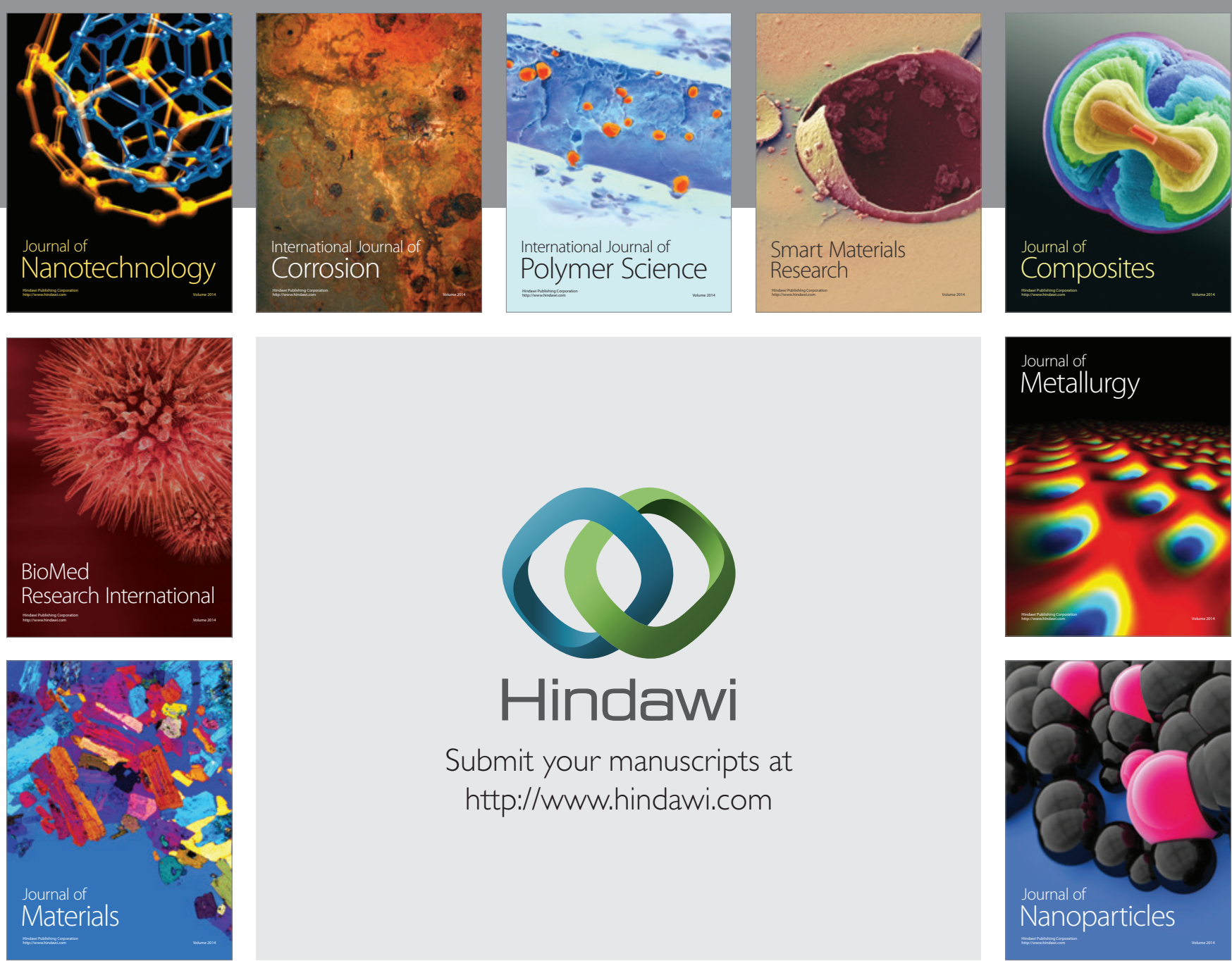

\section{Hindawi}

Submit your manuscripts at

http://www.hindawi.com

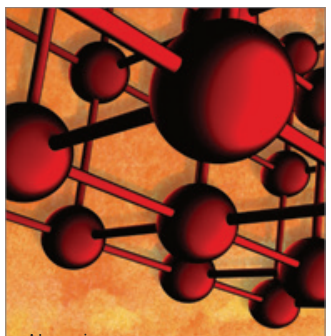

Materials Science and Engineering
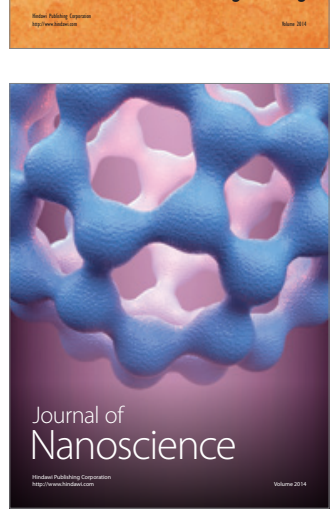
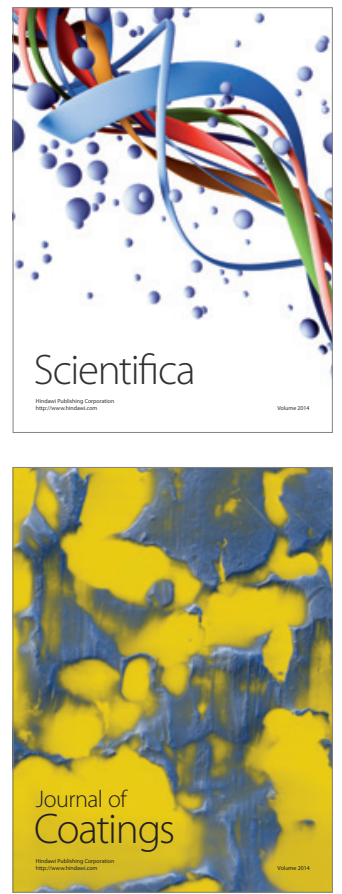
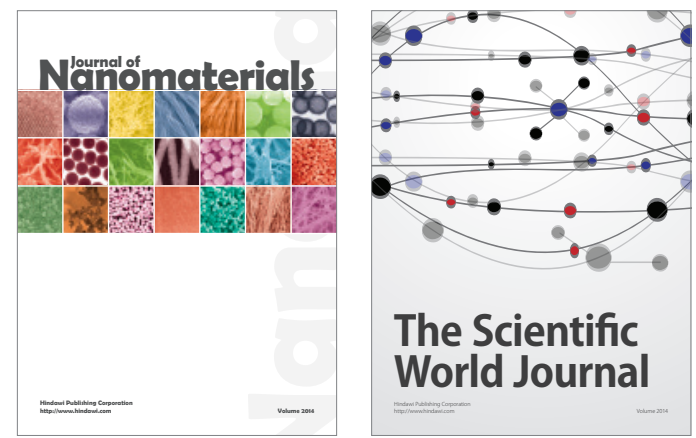

The Scientific World Journal
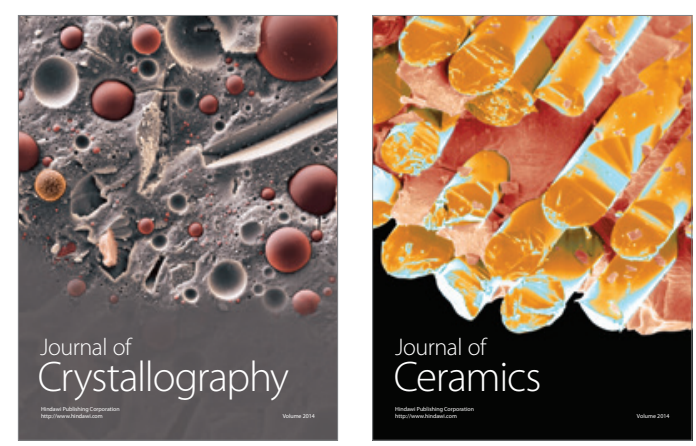
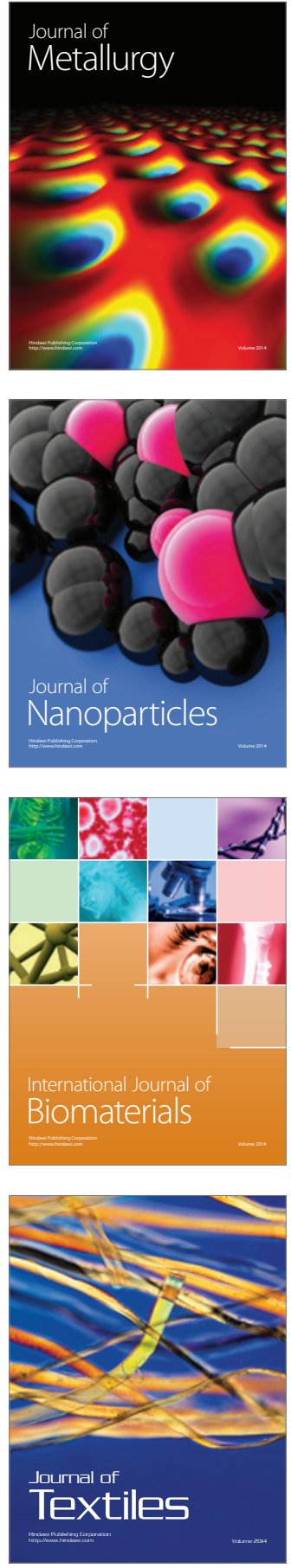